Artículo científico

Volumen 31(2):353-366. Mayo-agosto, 2020

e-ISSN 2215-3608, doi:10.15517/am.v31i2.38389

http://www.revistas.ucr.ac.cr/index.php/agromeso

\title{
Evaluación técnica-económica de la frecuencia de suplementación de novillos en pastoreo en Córdoba, Colombia ${ }^{1}$
}

\section{Technical-economic assessment of supplementation frequency over grazing steer in Córdoba, Colombia}

\author{
Lorena Inés Mestra-Vargas ${ }^{2}$, Wilson Andrés Barragán-Hernández ${ }^{2}$, Diego Andrés Medina-Herrera ${ }^{2}$, \\ Hernando Flórez-Díaz
}

1 Recepción: 22 de julio, 2019. Aceptación: 5 de diciembre, 2019. Esta Investigación se desarrolló en el marco del proyecto titulado "Estrategias para mejorar la competitividad y sostenibilidad de los sistemas de producción de leche y/o carne en el Caribe Húmedo", financiado por el Ministerio de Agricultura y Desarrollo Rural (MADR) y ejecutado por la Corporación Colombiana de Investigación Agropecuaria (AGROSAVIA) Convenio TV-15.

2 Corporación Colombiana de Investigación Agropecuaria (AGROSAVIA), Centro de Investigación Turipaná. Cereté, Colombia. 1mestra@ agrosavia.co (autor para correspondencia, https://orcid.org/0000-0003-3717-0153); wbarraganh@agrosavia.co (http://orcid.org/0000-00033528-4296); damedina@agrosavia.co (https://orcid.org/0000-0003-1761-9777).

3 Corporación Colombiana de Investigación Agropecuaria (AGROSAVIA), Centro de Investigación La Libertad. Villavicencio, Colombia. hflorez@agrosavia.co (https://orcid.org/0000-0002-0067-3315).

\section{Resumen}

Introducción. La producción de carne bovina se ve afectada por la estacionalidad forrajera y el costo de alimentación. La evaluación de diferentes frecuencias de alimentación en función de la pastura, podría sugerir una práctica apropiada para mejorar la rentabilidad. Objetivo. Evaluar técnica y económicamente la frecuencia de suplementación de novillos de ceba Brahman en pastoreo. Materiales y métodos. En el valle medio del Río Sinú, Colombia, se utilizaron 108 novillos distribuidos en un diseño de parcelas divididas en tres pasturas (Cynodon nlemfuensis, Megathyrsus maximus y Brachiaria hybrida, parcela principal) y tres esquemas de suplementación (subparcela): todos los días (STD), cada dos días (SDpM) y sin suplementación (SS). En el animal se evaluó la ganancia diaria de peso (GDP) y características morfométricas. En la canal: peso, rendimiento, longitud, perímetro de pierna, espesor de grasa e índice de compacidad. Los resultados biofísicos alimentaron la evaluación financiera de la tecnología implementada. Resultados. Hubo interacción entre la pastura y la frecuencia de suplementación $(\mathrm{p}<0,05)$ sobre la GDP. La mayor GDP se observó en animales de los tratamientos STD y en la gramínea C. nlemfuensis (680 g día $\mathrm{a}^{-1}$ ), mientras que con la pastura de $B$. hybrida cv Mulato II en SDpM se encontró la menor GDP (500 g día $\left.{ }^{-1}\right)$. Hubo efecto $(\mathrm{p}<0,05)$ de la dieta en la longitud corporal (150 vs $152 \mathrm{~cm}$ para novillos no suplementados y suplementados, respectivamente). No hubo diferencias $(\mathrm{p}<0,05)$ entre tratamientos, sobre las características de la canal. Conclusiones. La respuesta biológica en GDP no compensó el costo operativo diferencial en animales suplementados. Es necesario evaluar nuevas dietas con materias primas y niveles de inclusión que contribuyan al aumento de la producción animal, para generar una respuesta diferencial del beneficio técnico y económico de la tecnología.

Palabras claves: composición de la canal, biometría, forrajes, ganancia de peso, semilla de algodón, salvado de arroz. 


\begin{abstract}
Introduction. Beef production is affected by stationarity forage production and fed cost. Feed frequency evaluations according to pasture could suggest an appropriate practice for profitability improvement. Objective. To evaluate technically and economically the frequency of supplementation of Brahman fattening steers in grazing. Materials and methods. In the middle valley of the Sinú river, Colombia, 108 steers were used distributed in a splitplot design into three pastures (Cynodon nlemfuensis, Megathyrsus maximus, and Brachiaria hybrida, main plot) and three supplementation schemes (subplot): daily (STD), every two days (SDpM) and without supplementation (SS). The daily weight gain (GPD) and morphometric characteristics were evaluated in steers. In the carcass: weight, yield, length, leg perimeter, fat thickness, and compactness index. The biophysical data results fed the financial evaluation of the implemented technology. Results. An interaction of pasture and supplementation frequency over daily weight gain was detected $(\mathrm{p}<0.05)$. The highest GPD was observed in animals of the STD treatment for the grass C. nlemfuensis $\left(680 \mathrm{~g} \mathrm{day}^{-1}\right)$, while a $B$. hybrida cv Mulato II in SDpM recorded the lowest GPD with $500 \mathrm{~g}$ day ${ }^{-1}$. An effect $(\mathrm{p}<0.05)$ of the diet on body length was observed (150 vs. $152 \mathrm{~cm}$ for not supplemented and supplemented steers, respectively). There were no differences $(\mathrm{p}<0.05)$ between treatments over carcass characteristics. Conclusions. The biological response in GPD did not compensate for the differential operating cost in the supplemented animals. It is necessary to evaluate new diets with raw materials and inclusion levels that contribute to the increase in animal production, to generate a detectable change in the animal and economic response.
\end{abstract}

Keywords: carcass composition, biometrics, forages, weight gain, cottonseed, rice bran.

\title{
Introducción
}

Colombia es el tercer país en Suramérica con el mayor inventario de ganado bovino (28 millones de cabezas), superado por Brasil y Argentina (FAO, 2018). Esta información es consecuente con la importancia económica de la actividad ganadera en el país, la cual contribuye con 1,4\% del PIB nacional y genera el $6 \%$ del empleo nacional y $19 \%$ del pecuario (Fedegan, 2018). Sin embargo, pese a la representatividad del sector ganadero en la economía nacional y en el inventario suramericano, su productividad es baja comparada con los referentes del sector cárnico en Suramérica, contra los cuales Colombia presenta bajos índices de natalidad $(<60 \%)$, baja ganancia diaria de peso (298 $\mathrm{g} \mathrm{día}^{-1}$ ), elevada edad al sacrificio (>30 meses) y bajo rendimiento en canal (53\%) (García y Ruíz, 2016).

Se ha planteado que las problemáticas relacionadas a la baja eficiencia técnica-económica de la ganadería de carne en Colombia, se asocia con la fluctuación de la oferta y calidad de la alimentación, y a los elevados costos de producción (Santana et al., 2009). Bajo condiciones de la región Caribe colombiana, se han encontrado fluctuaciones del $60 \%$ de la producción de forraje y disminuciones de hasta $38 \%$ en la digestibilidad y $70 \%$ en la proteína cruda, entre los períodos de lluvia y sequía (Cuadrado et al., 2004; Cajas et al., 2012), lo cual representa pérdidas de peso vivo que oscilan entre 20 y $40 \mathrm{~kg}_{\text {animal }}{ }^{-1}$, con un importante impacto en la economía regional (Torregroza et al., 2015). Asimismo, Montoya et al. (2003) y Viloria (2003) han planteado que los costos operacionales en sistemas de producción de carne pueden variar entre $19 \%$ y $50 \%$ del costo total de producción, afectado directamente por los esquemas de suministro de alimento, principalmente en los periodos de sequía del año.

Dentro de las demandas de la cadena cárnica planteadas en el Plan Estratégico de Ciencia, Tecnología e Innovación (PECTIA), para la región Caribe de Colombia, se han planteado esquemas de trabajo relacionados con 
optimizar la edad al sacrificio y el manejo eficiente de los recursos animal y forrajero (García y Ruíz, 2016), lo cual está estrictamente ligado al incremento de la ganancia diaria de peso, mediante la optimización en los esquemas de alimentación. Estas demandas deben conducir a esquemas de intensificación sostenibles, preferiblemente basados en pasturas, dado el impacto del sistema ganadero en temas asociados al ambiente como el cambio y la variabilidad climática (Rao et al., 2015). Sin embargo, dado que existen pasturas que no podrían suplir la cantidad y calidad de nutrientes necesarios para ganancia de peso y al histórico registro del uso de tecnologías nutricionales energéticoproteicas y su repuesta en la producción ganadera (Makkar, 2011), se considera la inclusión de subproductos de la agroindustria como alternativa en la mejora de la calidad de la dieta.

El objetivo del presente trabajo fue evaluar técnica y económicamente la frecuencia de suplementación energético-proteica en novillos de ceba Brahman en pastoreo.

\section{Materiales y métodos}

\section{Área de estudio}

La investigación se llevó a cabo durante los meses de agosto de 2015 a mayo de 2016 en un área de 36 ha ubicada en el Centro de Investigación Turipaná, vinculado a la Corporación Colombiana de Investigación Agropecuaria (AGROSAVIA), con sede en el municipio de Cereté, Colombia, a 14 msnm, 8 50'79" N, 75 47'58" $\mathrm{O}$, con temperatura promedio de $28{ }^{\circ} \mathrm{C}$, humedad relativa de $87 \%$ y una precipitación promedio anual de 1200 mm, de acuerdo con información de la estación climática del Centro de Investigación Turipaná. Según Holdridge y Leslie (1971), el área se clasifica como bosque seco tropical.

El lote experimental se estableció en el año 2011, como parte de una investigación que evalúo la intensificación de la producción de carne vía fertirriego, para lo cual se establecieron dos bloques de 18 ha cada uno, con el objeto de controlar una condición diferencial de drenaje en el suelo. En cada bloque se establecieron de manera aleatoria 6 ha de las gramíneas Megathyrsus maximus (Jacq.) B.K. Simon \& S.W.L. Jacobs, Cynodon nlemfuensis Vanderyst y Brachiaria hybrida Basappa \& Muniy. Cv Mulato II. Cada área de 6 ha fue dividida en tres rotaciones independientes de ocho divisiones cada una $\left(2500 \mathrm{~m}^{2}\right)$, en las cuales se manejó una rotación de tres días de ocupación y 21 días de descanso (Reza-García et al., 2011). Durante el desarrollo del experimento se realizó una fertilización con base en el suministro de $23 \mathrm{~kg}_{\text {de }} \mathrm{N} \mathrm{ha}^{-1} \mathrm{año}^{-1}$.

\section{Descripción de los animales, suministro de dieta y diseño experimental}

Todos los procedimientos experimentales realizados en este estudio fueron aprobados por el Comité de Ética de la Corporación Colombiana de Investigación Agropecuaria (AGROSAVIA).

Se utilizaron 108 novillos Brahman comercial, aproximadamente de $22 \pm 5,0$ meses de edad y $336,7 \pm 11 \mathrm{~kg}$ de peso vivo. A cada tratamiento (pastura; parcela principal) se asignaron aleatoriamente 36 bovinos (tres repeticiones de doce bovinos cada una).

En cada una de las tres rotaciones asignadas en las pasturas $M$. maximus, $C$. nlemfuensis y $B$. hybrido cv. Mulato II, fue aleatorizada una frecuencia de suplementación de una mezcla de subproductos de la agroindustria (subparcela) correspondientes a: suplementación todos los días (STD), suplementación cada dos días (SDpM) y sin suplementación (SS). La suplementación con concentrado se realizó durante diecinueve semanas, con una semana de acostumbramiento. El suplemento ofrecido consistió en la mezcla de salvado de arroz (20 \%) y semilla 
de algodón (80\%), a razón de $1,5 \mathrm{~kg}^{\text {animal }}{ }^{-1}$ día $^{-1}\left(0,4 \%\right.$ del peso vivo) para el tratamiento STD, y $3 \mathrm{~kg}_{\text {animal }}{ }^{-1}$ cada dos días $(0,8 \%$ del peso vivo), para el tratamiento SDpM. Las proporciones de salvado de arroz y semilla de algodón se formularon con base en lo establecido por NRC (2016) para requerimientos nutricionales de bovinos de carne en fase de crecimiento y finalización. Durante todo el experimento, los animales recibieron sal mineralizada al $4 \%$ de fosforo $\left(80 \mathrm{~g} \mathrm{animal}^{-1}\right.$ día $\left.^{-1}\right)$ y agua a voluntad en bebederos automatizados instalados en los potreros. La composición nutricional de los forrajes y suplementos ofrecidos (Cuadro 1) se evaluó en el laboratorio de Nutrición animal del Centro de Investigación Turipaná (AGROSAVIA).

Cuadro 1. Composición nutricional de las pasturas y suplementos como porcentaje de la materia seca (MS). Centro de Investigación Turipaná, Corporación Colombiana de Investigación Agropecuaria (AGROSAVIA), municipio de Cereté, Colombia. 2016.

Table 1. Nutritional composition of grass and supplements as a dry matter percentage. Turipaná Research Center. Corporación Colombiana de Investigación Agropecuaria (AGROSAVIA). Cereté municipality, Colombia. 2016.

\begin{tabular}{lccccccc}
\hline Ítem & MS & Proteína & EE & FDNcp & FDA & Cnz & Dig. IS \\
\hline Estrella (C. nlemfuensis) & 25 & 10,6 & 2,6 & 62,8 & 34,8 & 10,2 & 55,71 \\
Mombasa (M. maximus) & 21 & 11,9 & 1,7 & 57,6 & 37,6 & 7,8 & 49,86 \\
Mulato II (Brachiaria hybrida) & 23 & 12,4 & 2,0 & 52,9 & 29,5 & 12,0 & 61,92 \\
Semilla de algodón (Gosypium sp.) & 92,2 & 23,6 & 20,4 & 35,0 & 27,8 & 4,3 & \\
Salvado de arroz (Oryza sativa L.) & 90 & 13,6 & 16 & 20,6 & 9,2 & 8,6 & \\
\hline
\end{tabular}

MS: materia seca; EE: extracto etéreo; FDNcp: fibra en detergente neutro corregida para cenizas y proteína; FDA: fibra en detergente ácido; Cnz: cenizas; Dig. IS: digestibilidad in situ de la materia seca / MS: dry matter; EE: ether extract; FDNcp: fiber in neutral detergent corrected for ash and protein; FDA: fiber in acid detergent; Cnz: ash; Dig. IS: in situ digestibility of dry matter.

La información se colectó con base en un diseño de parcelas divididas, conforme a la restricción en la aleatorización del factor pastura, sobre el cual se asignaron los tres niveles de frecuencia de alimentación.

\section{Disponibilidad y calidad composicional de las pasturas evaluadas}

La determinación de la disponibilidad de forraje en base seca $\left(\mathrm{kg} \mathrm{ha}^{-1}\right)$ se llevó a cabo mediante la metodología de disponibilidad por frecuencia, propuesta por Franco et al. (2006). La evaluación se realizó con base en cinco puntos de referencia asociados a la disponibilidad mínima (1) y máxima (5), sobre la cual se evaluó la composición y participación relativa de las especies en la composición botánica. Para determinar el porcentaje de materia seca en el forraje, se cortaron en campo $250 \mathrm{~g}$ de forraje verde y se pesaron con una balanza digital (capacidad de 5 $\pm 0,001$ $\mathrm{kg}$ ); posteriormente, la muestra se secó en estufa de ventilación forzada a $60^{\circ} \mathrm{C}$, de acuerdo con lo descrito en NTC4888 (ICONTEC, 2000) para determinar el valor de materia seca.

En cada pastura, se tomó una muestra representativa simulando pastoreo (hand plucking) para la determinación química de: cenizas (AOAC 942.05) proteína cruda (PC, AOAC 960.52 y 2001.11), fibra en detergente neutro (FDN, AOAC 2002.04), fibra en detergente ácido (FDA, AOAC 973.18) (Van-Soest et al., 1991), extracto etéreo (AOAC 973.18), según la AOAC (1995) y digestibilidad in situ de la materia seca (Ørskov et al., 1980). Todos los análisis se llevaron a cabo en el laboratorio de Nutrición Animal de AGROSAVIA Centro de Investigación Turipaná. 


\section{Ganancia de peso y medidas morfométricas}

El peso corporal vivo se determinó cada veintiocho días mediante una báscula electrónica (capacidad de $3000 \mathrm{~kg} \pm 0,1 \mathrm{~kg}$ ). Con base en el peso vivo, se determinó la ganancia diaria de peso acumulada en el período de evaluación, mediante la diferencia entre el peso inicial y final. Las variables morfométricas o de conformación, se determinaron cada 56 días, mediante el uso de bastón zoométrico y cinta métrica. En esta medición, se determinó la altura al sacro (distancia del suelo a las vértebras lumbar y sacra), perímetro torácico y abdominal (distancia del punto medio de cruz pasando por la línea del corazón y punto medio de vértebras lumbares pasando por la región inguinal, respectivamente), longitud corporal (distancia entre la tuberosidad isquiática y la articulación del encuentro), longitud de anca (distancia desde la tuberosidad coxal, hasta la tuberosidad isquiática) y amplitud de coxales e isquiones (distancia entre las tuberosidades coxales e isquiáticas, respectivamente).

\section{Evaluación en planta de beneficio}

Terminado el período experimental con un peso promedio de $470 \mathrm{~kg}$, los animales se sometieron a ayuno por $12 \mathrm{~h}$ y trasladados a una planta de beneficio privada (Red Cárnica-Minerva Foods), ubicada a $20 \mathrm{~km}$ del CI Turipaná. El beneficio se llevó a cabo con base en los protocolos sanitarios y de bienestar animal establecidos por la planta. A las 48 h después del sacrificio, siguiendo los métodos descritos por Amador et al. (1995), se evaluaron las siguientes variables de la canal: rendimiento de la canal caliente (cociente entre el peso de la canal caliente y el peso vivo), rendimiento de la canal fría (cociente entre la canal sometida a $4{ }^{\circ} \mathrm{C}$ por $48 \mathrm{~h}$ y el peso vivo) y longitud de la canal (LC), que correspondió a la medida entre el borde craneal de la sínfisis isquio-pubiana hasta el centro del borde craneal aparente de la primera costilla y el índice de compacidad de la canal, calculado como el cociente entre el peso de la canal fría $(\mathrm{kg})$ y la longitud de la canal $(\mathrm{cm})$ (García-Torres et al., 2005). En cada una de las canales también se determinó el grado de engrasamiento (GR) a través de la profundidad de los tejidos sobre la doceava costilla a $11 \mathrm{~cm}$ de la línea media, punto de grasa (Kirton y Johnson, 1979).

\section{Evaluación económica del modelo de producción de carne}

Con base en los coeficientes técnicos asociados a la ganancia de peso y kilogramos de carne producidos en las diferentes frecuencias de suplementación y pasturas evaluadas, se calcularon algunos indicadores de viabilidad financiera como costos unitarios y totales, ingresos brutos, ingresos netos y rentabilidad.

Costos: representan las salidas de dinero por concepto de compra de insumos para el manejo animal. Pueden ser fijos o variables. En estos se incluyó: mano de obra, insumos, equipos, alimentación y los indirectos como administración, impuestos y costos financieros. El costo operacional del manejo alimenticio se representó como el equivalente monetario de la cuantificación de tiempo dedicado a la actividad de alimentación (incluido el suministro de sal mineralizada), rotación y mantenimiento de potreros, mantenimiento de bebederos y manejo animal.

Ingreso neto: es la diferencia entre el ingreso bruto y el costo total. Indica si hay ganancia o pérdida (ecuación 1).

$$
I N=I B-C T \quad \text { ecuación } 1
$$

IN: ingreso neto.

IB: ingreso bruto.

CT: costos totales. 
Rentabilidad: es la relación entre el ingreso neto y el costo total, muestra los retornos o rendimientos de la inversión (ecuación 2):

$$
\text { Rentabilidad }=\frac{I N}{C T} * 100
$$

ecuación 2

\section{Análisis estadístico}

La información se analizó utilizando el paquete de análisis estadístico R Project versión 3.2.3, bajo un modelo de parcelas divididas, en el cual se consideró la pastura como parcela principal y la frecuencia de suplementación como subparcela. El modelo estadístico consideró como variables de respuesta las medidas de desempeño animal, morfométricas y de eficiencia en planta de beneficio, los efectos fijos de la parcela principal, subparcela e interacción entre estos factores y los efectos aleatorios bloque, error asociado a la parcela principal y el error experimental (ecuación 3).

$$
Y_{i j k}=\mu+P_{i}+D_{j}+P D_{i j}+B_{k}+P(B)_{i k}+e i j k \quad \text { ecuación } 3
$$

Donde:

$\mathrm{M}=$ media general.

$\mathrm{P}_{\mathrm{i}}=$ efecto de la i-esima pastura (Mombasa, Mulato II y Estrella - parcela principal).

$\mathrm{D}_{\mathrm{j}}=$ efecto de la j-esima frecuencia de suplementación (SS, SDpM y STD - subparcela).

$\mathrm{PD}_{\mathrm{ij}}=$ efecto de la interacción del efecto de la pastura y la frecuencia de suplementación.

$\mathrm{B}_{\mathrm{k}}=$ efecto del k-esimo bloque (1 y 2 ).

$\mathrm{P}(\mathrm{B})_{\mathrm{ik}}=$ error para la parcela principal. Id $\mathrm{N}\left(0, \sigma_{\mathrm{pp}}^{2}\right)$.

$\mathrm{e}_{\mathrm{ijk}}=$ error experimental. Id $\mathrm{N}\left(0, \sigma^{2}\right)$.

Las diferencias entre las medias de cuadrados mínimos en las variables debidas a los factores, se compararon mediante la prueba de Tukey al nivel de $\mathrm{p} \leq 0,05$.

\section{Resultados}

Se observó efecto $(\mathrm{p}<0,05)$ del pasto en la producción de forraje, del pasto e interacción dieta x pasto en la ganancia diaria de peso y de la dieta en la longitud corporal (Cuadro 2). La gramínea C. nlemfuensis presentó la mayor producción de forraje en $\mathrm{kg}$ de $\mathrm{MS} \mathrm{ha}^{-1}(1405 \mathrm{~kg}$ vs $872 \mathrm{~kg}$ para M. maximus y $841 \mathrm{~kg}$ para B. hybrida), lo cual fue consistente con la mayor ganancia de peso en el período de evaluación, con un registro que superó en $9,7 \%$ y $14,9 \%$ la ganancia observada en M. maximus y $B$. hybrido cv Mulato II, respectivamente, las cuales no registraron diferencias entre sí $(\mathrm{p}>0,05)$. La interacción dieta x pasto indicó que las mayores GDP se observaron en los tratamientos SDpM y STD para la gramínea C. nlemfuensis con un valor medio de $680 \mathrm{~g} \mathrm{día}^{-1}$, sin diferencia significativa de la GDP observadas en el tratamiento SS (610 $\left.\mathrm{g} \mathrm{día}^{-1}\right)$ para la misma pastura y en los tratamientos TD y SS en $B$. hybrida cv Mulato II y M. maximus, con GDP que variaron entre 580 y $620 \mathrm{~g} \mathrm{día}^{-1}$. El tratamiento SDpM en la pastura $B$. hybrida cv Mulato II, registró la GDP más baja, con un valor de $500 \mathrm{~g} \mathrm{día}^{-1}$, el cual presentó 
Cuadro 2. Ganancia diaria de peso y medidas morfométricas de novillos Brahman sometidos a pastoreo en gramíneas de los géneros Cynodon, Megathyrsus y Brachiaria, con tres frecuencias de suplementación. Centro de investigación Turipaná, Corporación Colombiana de Investigación Agropecuaria (AGROSAVIA), municipio de Cereté, Colombia. 2016.

Table 2. Daily weight gain and morphometric measurements of Brahman steers subjected to grazing in grasses of the Cynodon, Megathyrsus, and Brachiaria genera with three frequencies of supplementation. Turipaná Research Centre, Corporación Colombiana de Investigación Agropecuaria (AGROSAVIA). Cereté municipality, Colombia. 2016.

\begin{tabular}{|c|c|c|c|c|c|c|c|c|c|}
\hline & MS ha' ${ }^{-1}$ & GDP & APelv & PTor & PAbd & LCorp & LAnc & $A C o x$ & $A I s q$ \\
\hline & kg & g día ${ }^{-1}$ & \multicolumn{7}{|c|}{$\mathrm{Cm}$} \\
\hline \multicolumn{10}{|l|}{$\begin{array}{l}\text { Frecuencia de } \\
\text { suplementación }\end{array}$} \\
\hline SDpM & - & 600 & 139,74 & 185,94 & 211,70 & $152,48 \mathrm{a}$ & 50,25 & 42,32 & 18,09 \\
\hline SS & - & 601 & 138,25 & 183,80 & 212,81 & $150,10 \mathrm{~b}$ & 50,47 & 43,01 & 18,66 \\
\hline STD & - & 642 & 137,92 & 184,02 & 213,72 & $152,54 \mathrm{a}$ & 50,77 & 42,59 & 17,62 \\
\hline \multicolumn{10}{|l|}{ Pasto } \\
\hline C. nlemfuensis & $1405,9 \mathrm{a}$ & $660 \mathrm{a}$ & 139,44 & 185,41 & 214,68 & 152,98 & 51,08 & 42,84 & 18,08 \\
\hline M. maximus & $872,1 \mathrm{~b}$ & $604 \mathrm{~b}$ & 138,04 & 183,69 & 211,26 & 150,08 & 49,63 & 42,57 & 18,66 \\
\hline B. hybrido & $841,3 b$ & $576 \mathrm{~b}$ & 138,44 & 184,66 & 212,29 & 152,07 & 50,77 & 42,51 & 17,63 \\
\hline RCME & 389,92 & 0,121 & 4,657 & 6,696 & 6,243 & 4,109 & 1,996 & 1,406 & 1,418 \\
\hline \multicolumn{10}{|l|}{ Valor de p } \\
\hline Dieta & - & 0,0911 & 0,247 & 0,3701 & 0,8547 & 0,0132 & 0,5039 & 0,1343 & 0,105 \\
\hline Pasto & 0,0040 & 0,0009 & 0,7857 & 0,764 & 0,3924 & 0,1717 & 0,193 & 0,9509 & 0,1797 \\
\hline Dieta $x$ pasto & - & 0,0125 & 0,2765 & 0,8501 & 0,1083 & 0,0549 & 0,7677 & 0,0134 & 0,1493 \\
\hline
\end{tabular}

MS ha ${ }^{-1}$ : producción de materia seca por ha; GDP: ganancia diaria de peso; APelv: altura a la pelvis; PTor: perímetro torácico; PAbd: perímeto abdominal; LCorp: longitud corporal; LAnc: longitud de ancas; Acox: amplitud de coxales; AIsq: amplitud de isquiones; SDpM: Suplementación cada dos días; STD: Suplementación todos los días; SS: sin suplementación / MS ha-1 ${ }^{-1}$ dry matter yield per ha; GDP: daily weight gain; Apelv: height to the pelvis; PTor: thoracic perimeter; PAbd: abdominal girth; LCorp: body lenght; LAnc: lenght of haunches; Acox: coxal amplitude; Alsq: sit bone amplitudes; SDpM: every two days supplementation; STD: every day supplementation; SS: without supplementation.

${ }^{1}$ diciembre, enero y febrero constituyen período seco $/{ }^{1}$ December, January and February form the dry period.

Letras diferentes dentro de las columnas indican diferencias significativas según Tukey a una probabilidad $\mathrm{p}<0,05 /$ Different letters within the columns indicate significant differences according to Tukey at a probability $(\mathrm{p}<0.05)$.

diferencia significativa de todos los registros obtenidos en los tratamientos STD en las tres pasturas evaluadas y para el tratamiento SDpM en las gramíneas C. nlemfuensis y M. maximus (Figura 1).

Con relación a la longitud corporal, los animales suplementados superaron significativamente $(\mathrm{p}<0,05)$ a los animales no suplementados (152 vs $150 \mathrm{~cm}$, respectivamente). Las características de la canal no fueron influenciadas por los factores evaluados (Cuadro 3).

En todas las dietas y pasturas, los ingresos netos representaron entre el $31 \%$ y el $52 \%$ del costo total, por lo cual se deduce que la inversión fue viable desde el punto de vista económico (Cuadro 4). Sin embargo, un análisis más específico, evidenció que los costos operacionales que implican la suplementación en campo, los cuales 


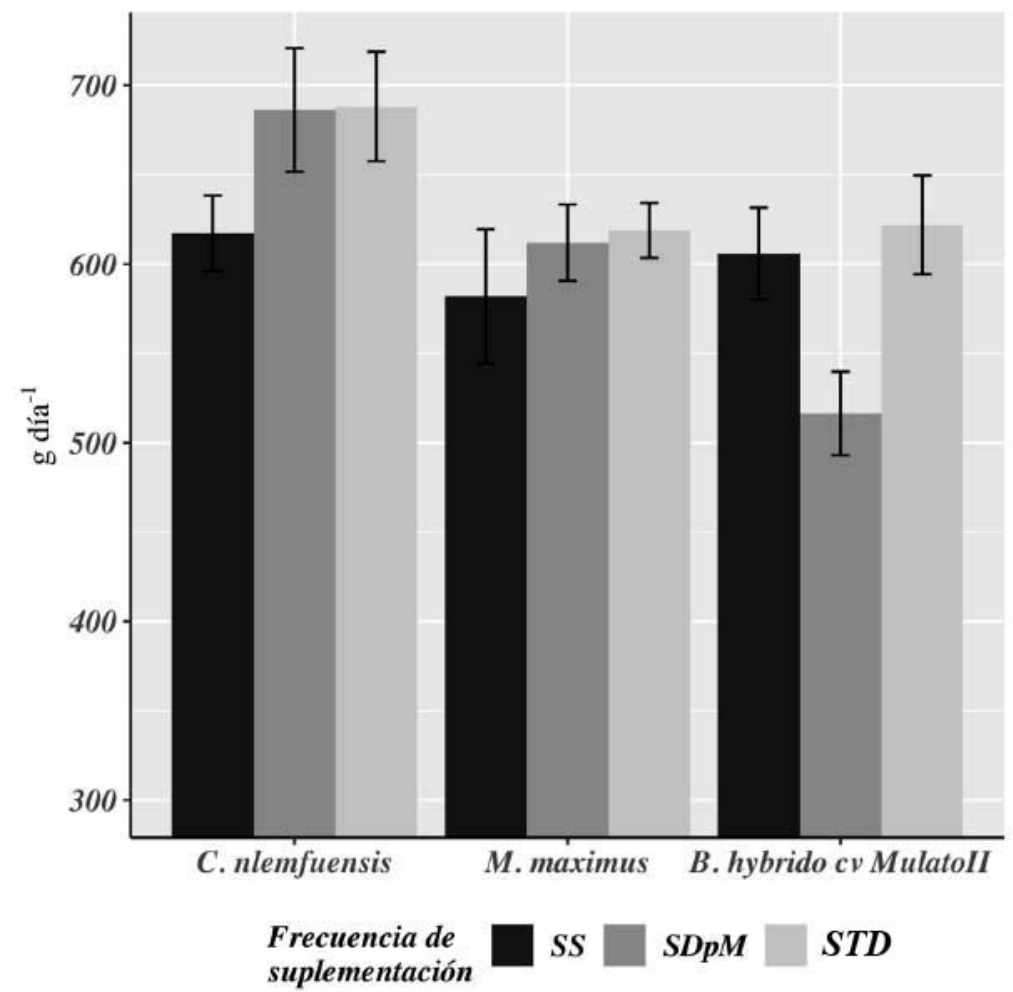

Figura 1. Efecto de la interacción frecuencia de suplementación x pastura para la ganancia de peso de novillos Brahman. Centro de investigación Turipaná, Corporación Colombiana de Investigación Agropecuaria (AGROSAVIA), municipio de Cereté, Colombia. 2016.

SDpM: suplementación cada dos días; STD: suplementación todos los días; SS: sin suplementación.

Barras de error corresponden al error estándar de la media.

Figure 1. Daily gain weight by supplementation frequency and pasture interaction in Brahman steers. Turipaná Research Center, Corporación Colombiana de Investigación Agropecuaria (AGROSAVIA). Cereté municipality, Colombia. 2016.

SDpM: every two days supplementation; STD: everyday supplementation; SS without supplementation.

Error bars are the standard error of the mean.

representan el $31 \%$ y el $66 \%$ para los tratamientos SDpM y STD, respectivamente, comparado con el costo base de operación en el tratamiento SS, no son compensados por la eficiencia biológica en ganancia de peso de los animales sometidos a las dietas, a pesar de observar los mejores desempeños en GDP, específicamente para la gramínea $C$. nlemfuensis. Lo anterior muestra que a menores costos de operación en el tratamiento SS se evidenció la mayor rentabilidad en el negocio, con reducciones de $5 \%$ y $12 \%$ en rentabilidad en C. nlemfuensis, $7 \%$ y $10 \%$ en $M$. maximus y $11 \%$ y $17 \%$ en B. hybrido, para los tratamientos SDpM y STD, respectivamente. 
Cuadro 3. Características de la canal de novillos Brahman sometidos a pastoreo en gramíneas de los géneros Cynodon, Megathyrsus y Brachiaria, con tres frecuencias de suplementación. Centro de investigación Turipaná, Corporación Colombiana de Investigación Agropecuaria (AGROSAVIA), municipio de Cereté, Colombia. 2016.

Table 3. Carcass traits of Brahman steers subjected to grazing in grasses of the genera Cynodon, Megathyrsus, and Brachiaria, with three frequencies of supplementation. Turipaná Research Center, Corporación Colombiana de Investigación Agropecuaria (AGROSAVIA). Cereté, municipality, Colombia. 2016.

\begin{tabular}{|c|c|c|c|c|c|c|c|c|c|}
\hline & PCC & PCF & RCC & RCF & LC & PP & G1 & G2 & IC \\
\hline & \multicolumn{2}{|c|}{ kg } & \multicolumn{2}{|c|}{$\%$} & \multicolumn{4}{|c|}{$\mathbf{c m}$} & $\mathrm{cm} \mathrm{kg}^{-1}$ \\
\hline \multicolumn{10}{|l|}{$\begin{array}{l}\text { Frecuencia de } \\
\text { suplementación }\end{array}$} \\
\hline SDpM & 257,71 & 250,88 & 54,63 & 53,19 & 143,84 & 83,37 & 1,30 & 1,07 & 1,79 \\
\hline SS & 254,98 & 248,17 & 54,59 & 53,13 & 143,16 & 82,88 & 1,15 & 1,24 & 1,78 \\
\hline STD & 261,88 & 255,03 & 55,03 & 53,59 & 145,15 & 83,44 & 1,31 & 1,24 & 1,80 \\
\hline \multicolumn{10}{|l|}{ Pasto } \\
\hline C. nlemfuensis & 259,34 & 252,37 & 54,51 & 53,05 & 144,50 & 84,12 & 1,23 & 1,30 & 1,79 \\
\hline M. maximus & 260,85 & 253,59 & 55,47 & 53,92 & 143,47 & 84,29 & 1,33 & 1,22 & 1,82 \\
\hline B. hybrido & 254,39 & 248,12 & 54,27 & 52,94 & 144,17 & 81,28 & 1,20 & 1,03 & 1,77 \\
\hline RCME & 13,241 & 12,900 & 1,698 & 1,651 & 3,348 & 2,802 & 0,377 & 0,493 & 0,088 \\
\hline \multicolumn{10}{|l|}{ Valor de p } \\
\hline Dieta & 0,2296 & 0,2166 & 0,5283 & 0,4798 & 0,14 & 0,701 & 0,16 & 0,5141 & 0,6384 \\
\hline Pasto & 0,8072 & 0,8387 & 0,6358 & 0,6561 & 0,464 & 0,4981 & 0,49 & 0,0848 & 0,7500 \\
\hline Trat $x$ Pasto & 0,6105 & 0,5827 & 0,1477 & 0,1177 & 0,518 & 0,8152 & 0,28 & 0,1148 & 0,2773 \\
\hline
\end{tabular}

PCC: peso de la canal caliente; PCF: peso de la canal fría; RCC: rendimiento de la canal caliente; RCF: rendimiento de la canal fría; LC: longitud de la canal; PP: perímetro de la pierna; G1 y G2: espesor de grasa; IC: índice de compasidad; SDpM: suplementación cada dos días; STD: suplementación todos los días; SS: sin suplementación / PCC: hot carcass weight; PCF: cold carcass weight; RCC: hot carcass yield; RCF: cold carcass yield; LC: carcass length; PP: shank perimeter; G1y G2: fat thickness; IC: compactness index; SDpM: every two days supplementation; STD: every day supplementation; SS without supplementation.

\section{Discusión}

La respuesta a esquemas de suplementación en energía y proteína bajo pastoreo depende de las características químicas y estructurales de la pastura base (Drewnoski et al., 2011), aspecto que pudo haber influenciado la mayor ganancia diaria de peso con la gramínea C.nlemfuensis para los novillos con suplementación, asociadas a su mayor disponibilidad de forraje. Se ha demostrado que la eficiencia productiva de la suplementación en pastoreo se basa en el aporte de fuentes de energía y proteína en rumen que favorecen la degradabilidad y tasa de pasaje como un efecto dinamizador de la capacidad ruminal para degradar la fibra (Costa et al., 2015). Sin embargo, esta eficiencia está condicionada a la disponibilidad de forraje y a la posibilidad de tener un óptimo consumo de materia seca (de-Medeiros et al., 2015). Algunos investigadores han evidenciado que en sistemas de pastoreo, la cantidad de 
Cuadro 4. Coeficientes técnicos e indicadores económicos de viabilidad para novillos Brahman sometidos a pastoreo en gramíneas de los géneros Cynodon, Megathyrsus y Brachiaria, con tres frecuencias de suplementación. Centro de investigación Turipaná, Corporación Colombiana de Investigación Agropecuaria (AGROSAVIA), municipio de Cereté, Colombia. 2016.

Table 4. Technical coefficients and economic indicators of viability for Brahman steers subjected of the Cynodon, Megathyrsus, and Brachiaria with three frequencies of supplementation. Turipaná Research Center, Corporación Colombiana de Investigación Agropecuaria, AGROSAVIA. Cereté municipality, Colombia. 2016.

\begin{tabular}{|c|c|c|c|c|c|c|c|c|c|}
\hline & \multicolumn{3}{|c|}{ Cynodon nlemnfunsis } & \multicolumn{3}{|c|}{ Megathyrsus maximus } & \multicolumn{3}{|c|}{ Brachiaria hybrido } \\
\hline & SS & SDpM & STD & SS & SDxM & STD & SS & SDpM & STD \\
\hline Peso inicial (kg) & 331,5 & 335,6 & 329,5 & 337,5 & 332,9 & 334,1 & 337,2 & 336,5 & 341,0 \\
\hline Peso final (kg) & 482,0 & 503,3 & 497,0 & 480,0 & 482,0 & 485,0 & 484,0 & 464,0 & 484,0 \\
\hline Rend. canal (\%) & 54,8 & 54,1 & 54,6 & 55,1 & 54,9 & 56,4 & 53,9 & 54,9 & 54,1 \\
\hline \multirow[t]{2}{*}{ Prod. carne ha ${ }^{-1} a_{\tilde{n}}{ }^{-1}$} & 675,8 & 751,5 & 750,9 & 637,3 & 670,2 & 677,6 & 663,4 & 550,1 & 681,0 \\
\hline & \multicolumn{9}{|c|}{ Miles de pesos \$ COP(USD*) } \\
\hline $\begin{array}{l}\text { Costo compra } \\
\text { animales }^{2}\end{array}$ & $\begin{array}{c}1209,3 \\
(354,34)\end{array}$ & $\begin{array}{c}1262,5 \\
(376,13)\end{array}$ & $\begin{array}{c}1227,6 \\
(364,87)\end{array}$ & $\begin{array}{c}1276,7 \\
(379,46)\end{array}$ & $\begin{array}{c}1235,4 \\
(367,19)\end{array}$ & $\begin{array}{c}1233,8 \\
(366,71)\end{array}$ & $\begin{array}{c}1247,6 \\
(370,81)\end{array}$ & $\begin{array}{c}1283,3 \\
(381,42)\end{array}$ & $\begin{array}{c}1229,6 \\
(365,46)\end{array}$ \\
\hline Costo operacional ${ }^{3}$ & $\begin{array}{c}283,3 \\
(84,20)\end{array}$ & $\begin{array}{c}371,9 \\
(110,54)\end{array}$ & $\begin{array}{c}460,5 \\
(136,87)\end{array}$ & $\begin{array}{c}283,3 \\
(84,20)\end{array}$ & $\begin{array}{c}371,9 \\
(110,54)\end{array}$ & $\begin{array}{c}460,5 \\
(136,87)\end{array}$ & $\begin{array}{c}283,3 \\
(84,20)\end{array}$ & $\begin{array}{c}371,9 \\
(110,54)\end{array}$ & $\begin{array}{c}470,2 \\
(136,87)\end{array}$ \\
\hline $\begin{array}{l}\text { Costo kg en planta de } \\
\text { beneficio }^{4}\end{array}$ & $\begin{array}{c}4,8 \\
(1,43)\end{array}$ & $\begin{array}{c}4,7 \\
(1,40)\end{array}$ & $\begin{array}{c}4,7 \\
(1,40)\end{array}$ & $\begin{array}{c}4,8 \\
(1,43)\end{array}$ & $\begin{array}{c}4,8 \\
(1,43)\end{array}$ & $\begin{array}{c}4,9 \\
(1,46)\end{array}$ & $\begin{array}{c}4,7 \\
(1,40)\end{array}$ & $\begin{array}{c}4,8 \\
(1,43)\end{array}$ & $\begin{array}{c}4,7 \\
(1,40)\end{array}$ \\
\hline Costo total & $\begin{array}{c}1492,6 \\
(443,63)\end{array}$ & $\begin{array}{c}1634,4 \\
(485,78)\end{array}$ & $\begin{array}{c}1688,1 \\
(501,74)\end{array}$ & $\begin{array}{c}1560,0 \\
(463,66)\end{array}$ & $\begin{array}{c}1607,3 \\
(477,72)\end{array}$ & $\begin{array}{c}1694,3 \\
(503,58)\end{array}$ & $\begin{array}{c}1530,9 \\
(455,02)\end{array}$ & $\begin{array}{c}1655,2 \\
(491,96)\end{array}$ & $\begin{array}{c}1699,9 \\
(505.25)\end{array}$ \\
\hline Ingreso bruto & $\begin{array}{c}2292,0 \\
(681,23)\end{array}$ & $\begin{array}{c}2365,0 \\
(702,93)\end{array}$ & $\begin{array}{c}2354,1 \\
(699,69)\end{array}$ & $\begin{array}{c}2296,7 \\
(682,63)\end{array}$ & $\begin{array}{c}2295,6 \\
(682,30)\end{array}$ & $\begin{array}{c}2374,1 \\
(705,63)\end{array}$ & $\begin{array}{c}2262,4 \\
(672,43)\end{array}$ & $\begin{array}{c}2210,9 \\
(657,13)\end{array}$ & $\begin{array}{c}2271,8 \\
(675,23)\end{array}$ \\
\hline Ingreso neto & $\begin{array}{c}782,9 \\
(232,69)\end{array}$ & $\begin{array}{c}751,3 \\
(223.30)\end{array}$ & $\begin{array}{c}675,3 \\
(200,71)\end{array}$ & $\begin{array}{c}763,9 \\
(227,05)\end{array}$ & $\begin{array}{c}691,9 \\
(205,65)\end{array}$ & $\begin{array}{c}678,3 \\
(201,60)\end{array}$ & $\begin{array}{c}731,5 \\
(217,42)\end{array}$ & $\begin{array}{c}593,7 \\
(176,46)\end{array}$ & $\begin{array}{c}539,4 \\
(160,32)\end{array}$ \\
\hline Rentabilidad (\%) & 52 & 47 & 40 & 50 & 43 & 40 & 48 & 37 & 31 \\
\hline
\end{tabular}

1: estimada a partir de la ganancia diaria de peso acumulada (Cuadro 2) y la capacidad de carga (3 animales ha-1) / Estimated from the accumulated daily weight gain (Table 2$)$ and the stocking rate $\left(3\right.$ animals ha $\left.{ }^{-1}\right)$.

2: precio de compra $\$ 3700$ pesos COP / Purchase price $\$ 3700$ pesos COP.

3: costo de mano de obra y alimentación, manejo de la rotación y mantenimiento de potreros / Cost of labor and feeding, management of rotation and maintenance of paddocks.

4: costo de kilogramo en pie en la planta de beneficio. Precio base de $\$ 4600$ pesos COP, con bonificación por rendimiento en canal por encima de $51 \%$ / Kilogram cost standing in the processing plant. Base price of $\$ 4600$ pesos COP, with bonification for dressing above $51 \%$. * \$1 USD equivale a \$3354,50 COP (Tasa de cambio oficial; 30 mayo 2019) / \$1 USD equals to \$3354.50 COP (Official exchange rate; May 30, 2019).

SDpM: suplementación cada dos días; STD: suplementación todos los días; SS: sin suplementación / SDpM: s every two days supplementation; STD: every day supplementation; SS: without supplementation.

alimento consumido es el principal factor que determina la productividad animal (Burns y Sollenberger, 2002) y es el resultado de las decisiones del animal en una escala temporal y espacial de múltiples estratos (Senft et al., 1987; Bailey et al., 1996; Kolver y Muller, 1998) que resulta del proceso de pastoreo (Gibb, 1998).

La ausencia de diferencias significativas en la respuesta productiva entre las frecuencias de suplementación ha sido documentada en la literatura; en ese sentido, Moriel et al. (2012) no encontraron diferencias significativas 
al comparar una frecuencia de suplementación diaria $\left(270 \mathrm{~g}\right.$ día $\left.^{-1}\right)$, contra cada tres días ( $\left.250 \mathrm{~g}_{\text {día }}{ }^{-1}\right)$ en novillas cruzadas; Brahman x raza Británica en pasturas de baja (C. nlemfuensis) y mediana calidad (Cynodon dactylon); al igual que Franco et al. (2018), quienes reportaron ganancias diarias de peso $(p>0,05)$ para suplementaciones diaria, cada tres días y una vez por semana de 177, 183 y $190 \mathrm{~g} \mathrm{día}^{-1}$, respectivamente, en novillos Nelore de 174 $\mathrm{kg}$, alimentados en pastoreo de Urochloa brizantha cv Marandú, y Bonadimann et al. (2017), reportaron igual ganancia diaria de peso al comparar suplementación tres veces por semana, contra todos los días (1000 vs 1020 g día ${ }^{-1}$, respectivamente) en bovinos de carne bajo pastoreo de $U$. brizantha cv Maradú. Esta condición puede ser explicada por la eficiencia en el uso del nitrógeno, la cual se ha indicado que, en dietas suministradas hasta tres veces por semana, no afecta la disponibilidad de compuestos nitrogenados en el rumen o la eficiencia de su uso (de-Moraes et al., 2010).

En el presente trabajo no se evidenciaron diferencias significativas en la ganancia diaria de peso entre dietas. Contrario a esto, de-Figueiredo (2015) y Franco et al. (2018) indicaron que las frecuencias de suplementación evaluadas, diaria o una vez por semana, mejoraban la ganancia diaria de peso, lo que probablemente pudo ser atribuido a suministro de nutrientes en la dieta, el cual fue de $26 \%$ de PC y $84 \%$ de TDN y $22 \%$ de PC con $88 \%$ de TDN, respectivamente, los cuales están por encima de los aportes nutricionales en las dietas SDpM y STD con 13 y $14,3 \%$ de PC y 57 y $67 \%$ de TDN usadas en el presente trabajo.

En la literatura se registran trabajos que asocian la frecuencia de suplementación con el desempeño productivo (principalmente ganancia de peso), pero no con el desarrollo corporal del animal (de-Paula et al., 2010; Moriel et al., 2012; de-Figueiredo et al., 2015). El presente trabajo reveló una asociación de la frecuencia de suplementación con la longitud del animal. Los resultados obtenidos en este estudio para los novillos suplementados, fueron superiores a los obtenidos por Velásquez y Álvarez (2004), en novillos Brahman de 24 meses de edad, con una media de $148 \mathrm{~cm}$ y a la media de longitud corporal registrada para una población de 2590 animales Brahman de 13 meses de edad de 120,5 cm, y de 20 meses de edad con un valor de 129,3 cm (Kamprasert et al., 2019). Con relación a los rendimientos de la canal, en todos los casos se observó un rendimiento en canal caliente (RCC) y peso de la canal fría (PCF), que superó la media nacional reportada por Santana et al. (2009), establecida en $52 \%$ y $205 \mathrm{~kg}$, respectivamente.

Varios autores han relacionado el efecto positivo de reducir la frecuencia de suplementación sobre los costos operativos en sistemas de producción de carne, como una consecuencia directa del menor gasto en mano de obra, combustible, horas de trabajo en máquina, entre otros (de-Paula et al., 2010; de-Figueiredo et al., 2015; Bonadimann et al., 2017). En el presente trabajo no se registró una ventaja comparativa entre las frecuencias de suplementación y el tratamiento sin suplementación, probablemente motivado por la poca respuesta biológica en ganancia de peso asociada a las dietas, las cuales no lograron compensar el costo incremental en el suministro del alimento y a la calidad nutricional de las pasturas evaluadas. Al igual que en el presente trabajo, Sossa y Barahona (2015), en condiciones de trópico de altura registraron rentabilidades de $34 \%$ y $33 \%$ para la respuesta productiva de novillos en pastoreo en trópico de altura con y sin suplementación energética, respectivamente, argumentando que la sola pastura podría permitir una respuesta productiva rentable. Para trópico bajo, Torregroza et al. (2004) reportaron rentabilidad de 45,9\% en animales cebú comerciales suplementados con $5 \mathrm{~kg}$ de semilla de algodón durante el período seco, en un modelo de producción de una tonelada de carne por hectárea año ${ }^{-1}$, sin presentar información para animales sin suplementar. En similar condición a los anteriores autores, Montoya et al. (2003) registraron rentabilidades 19,7 \% en el periodo lluvioso y 33,6 \% en el período seco, en un sistema de producción de carne en el Valle del Sinú (Córdoba - Colombia). 


\section{Conclusiones}

La pastura $C$. nlemfuensis con o sin suplementación, registró un comportamiento biológicamente superior en la ganancia diaria de peso comparado con los demás tratamientos. Sin embargo, esta ventaja no se reflejó en una mayor rentabilidad económica, pero redujo el ciclo de la ceba de animales en doce meses, lo cual puede representar una reducción en costos operativos y rápida recuperación de la inversión por concepto de la venta de semovientes al frigorífico. El rendimiento de las canales en este tratamiento superó el promedio nacional de $52 \%$.

Al no registrarse cambios en las medidas morfológicas de los animales y en las variables evaluadas en la canal, es necesario implementar un esquema de suplementación y balance nutricional con materias primas que puedan generar una respuesta biológica capaz de compensar el gasto operativo que implica la suplementación, con el objeto de generar una mayor rentabilidad.

El análisis económico realizado en el presente trabajo no permite extrapolar los resultados a condiciones diferentes a las cuales se consideraron para desarrollar este experimento, por lo cual se considera necesario un abordaje económico más exhaustivo en un entorno biofísico de mayor representatividad en el sistema de producción de carne.

\section{Agradecimientos}

Los autores agradecen a la Corporación Colombiana de Investigación Agropecuaria (AGROSAVIA), por el soporte logístico y financiero en la ejecución de esta investigación.

\section{Literatura citada}

Amador, I., A. Palacios, y M. Maldonado. 1995. Sistema ICTA de Clasificacion de Canales y Cortes de Carne Bovina. Ministerio de Agricultura y Desarrollo Rural, Universidad Nacional de Colombia, y Servicio Nacional de, Bogotá, COL.

AOAC (Association of Official Analytical Chemist). 1995. Official methods of analysis. AOAC Int., VA, USA.

Bailey, D.W., J.E. Gross, E.A. Laca, L.R. Rittenhouse, M.B. Coughenour, D.M. Swift, and P.L. Sims. 1996. Mechanisms that result in large herbivore grazing distribution patterns. J. Range Manage. 49:386-400. doi:10.2307/4002919

Bonadimann, R., E.H.B.K. de-Moraes, K.A.K. de-Moraes, D.S. Pina, A.S. de-Oliveira, D.M. de-Meneses, H. Stinguel, and C.M. de-Almeida. 2017. Frequency of concentrate supplementation for grazing beef cattle. Sem. Cienc. Agrar. 38:2141-2150. doi:10.5433/1679-0359.2017v38n4p2141

Burns, J.C., and L.E. Sollenberger. 2002. Grazing behavior of ruminants and daily performance from warm-season grasses. Crop Sci. 42:873-881. doi:10.2135/cropsci2002.8730

Cajas, Y., W. Barragán, L. Arreaza, J. Argüelles, E. Amézquita, Y. Abuabara, y C. Lascano. 2012. Efecto sobre la producción de carne de la aplicación de tecnologías de renovación de praderas de Bothriochloa pertusa (L.) A. Camus en la Costa Norte Colombiana. CORPOICA Cienc. Tecnol. Agropecu. 13:213-218.

Costa, N.L., A.L.G. Monteiro, A.L.P. Silva, A. de-Moraes, A.F. Giostri, T.S.S. Stivari, T.C. Baldissera, e E.A. Pin. 2015. Considerações sobre a degradação da fibra em forragens tropicais associada com suplementos energéticos ou nitrogenados. Arch. Zootec. 64:31-41. doi:10.21071/az.v64i247.504

Cuadrado, H., L. Torregroza, y N. Jiménez. 2004. Comparación bajo pastoreo con bovinos machos de ceba de cuatro especies de gramíneas del género Brachiaria. Rev. MVZ Córdoba 9:438-443. doi:10.21897/rmvz.485 
de-Figueiredo, L.V., J. Tilemahos, L. Silva, L.K. Hatamoto-Zervoudakis, P. Veiga, E.H. de-Moraes, e J.F. Koscheck. 2015. Proteína degradável no rúmen e frequência de suplementação para novilhos Nelore em pastejo: Desempenho produtivo e análise econômica. Sem. Ciênc. Agrár. 36:2105-2118. doi:10.5433/1679-0359.2015v36n3Supl1p2105

de-Medeiros, S.R., R.C. Gomes, e D.J. Bungenstab. 2015. Nutrição de bovinos de corte: Fundamentos e aplicações. Embrapa, Brasilia D.F., BRA. https://ainfo.cnptia.embrapa.br/digital/bitstream/item/120040/1/Nutricao-Animal-livro-em-baixa.pdf (consultado 28 fev. 2019).

de-Moraes, E.H.B.K., M.F. Paulino, S.C.V. Filho, S., K.A.K. de-Moraes, E. Detmann, e M.G. de-Souza. 2010. Avaliação nutricional de estratégias de suplementação para bovinos de corte durante a estação da seca. R. Bras. Zootec. 39:608-616. doi:10.1590/S1516-35982010000300021

de-Paula, N.F., J.T. Zervoudakis, L.S. Cabral, D.M.G. de-Carvalho, L.K. Htamoto-Zervoudakis, H.B.K. de Moraes, e A.A. de-Oliveira. 2010. Frequência de suplementação e fontes de proteína para recria de bovinos em pastejo no período seco: desempenho produtivo e econômico. R. Bras. Zootec. 34:873-882. doi:10.1590/S1516-35982010000400024

Drewnoski, M.E., M.H. Poore, and G.A. Benson. 2011. Effect of frequency of supplementation of a soyhulls and corn gluten feed blend on hay intake and performance of growing steers. Anim. Feed Sci. Technol. 164:38-44. doi:10.1007/s12264-018-0248-0

FAO. 2018. FAOSTAT Base de datos de inventario en ganadería vacuna para América Latina. FAO, Roma, ITA. http://www. fao.org/faostat/es/\#data/QA (consultado 10 jul. 2019).

Fedegan (Federación Colombiana de Ganaderos). 2018. Cifras de referencia sector ganadero colombiano 2018. Fedegan, Bogotá, COL.

Franco, L.H., D. Calero, y C.V. Durán. 2006. Manejo y utilización de forrajes tropicales multipropósitos. Universidad Nacional de Colombia, Palmira, COL. http://bdigital.unal.edu.co/5052/1/9789584411754.pdf (consultado 15 ene. 2019).

Franco, G.L., M. Vedovatto, M.C. D’Oliveira, I.M.C. Neto, M. G. Morais, e J.M.S. Diogo. 2018. Frequência de suplementação proteico-energética sobre o desempenho e comportamento ingestivo de bezerros Nelore mantidos em pastagem tropical na estação seca. Sem. Ciênc. Agrár. 39:2555-2564. doi:10.5433/1679-0359.2018v39n6p2555

García-Torres, S., M. Espejo, M. López, M. Izquierdo, J. Mendizábal, y A. Purroy. 2005. Conformación, engrasamiento y sistemas de clasificación de la canal bovina. En: V. Cañeque, y C. Sañudo, editores, Estandarización de las metodologías para evaluar la calidad del producto (animal vivo, canal, carne y grasa) en los rumiantes. Instituto Nacional de Investigación y Tecnología Agraria y Alimentaria, Madrid, ESP. p. 105-119.

García, G., y D. Ruíz. 2016. Plan estratégico de ciencia, tecnología e innovación del sector agropecuario colombiano. Cadena cárnica bovina. Anexo 5. Colombia Competitiva, COL. http://www.colombiacompetitiva.gov.co/sncei/Documents/pectiaterminado.pdf (consultado 10 jul. 2019).

Gibb, M.J. 1998. Animal grazing/intake terminology and definitions. En: Keane M.G. and E.G. O'Riordan, editors, $8^{\text {th }}$ European Intake Workshop. Proceedings of pasture ecology and animal intake groups join meeting. St. Patrick's College, Dublin, IRL. p. 21-35.

Holdridge, L.R., and R. Leslie. 1971. Forest environments in tropical life zones; a pilot study. Pergamon Press, Oxford, NY, USA.

ICONTEC (Instituto Colombiano de Normas Técnicas y Certificación). 2000. Norma Técnica Colombiana. NTC 4888. Alimentos para animales. Determinación del contenido de humedad y materia volátil. ICONTEC, Bogotá, COL.

Kamprasert, N., N. Duijvesteijn, and J.H.J. Van-der-Werf. 2019. Estimation of genetic parameters for BW and body measurements in Brahman cattle. Animal doi:10.1017/S1751731118003348

Kirton, A.H., and D.L. Johnson. 1979. Interrelationships between GR and other lamb carcass fatness measurements. Proc. New Zealand Soc. Anim. Prod. 39:194-201. 
Kolver, E.S., and L.D. Muller. 1998. Performance and nutrient intake of high producing Holstein cows consuming pasture or a total mixed ration. J. Dairy Sci. 81:1403-1411. doi:10.3168/jds.S0022-0302(98)75704-2

Makkar, H. 2011. Successes and failures with animal nutrition practices and technologies in developing countries. FAO, ROMA, ITA. http://www.fao.org/docrep/014/i2270e/i2270e00.pdf (accessed Mar. 5, 2019).

Montoya, J., L. Torregroza, M. Palomino, M. González, H. Cuadrado, S. Reza, y U. Gómez. 2003. Análisis técnico y económico de un modelo de producción de carne en el Valle del Sinú. Rev. MVZ Córdoba 8:265-272. doi:10.21897/rmvz.1051

Moriel, P., R.F. Cooke, D.W. Bohnert, J.M.B. Vendramini, and J.D. Arthington. 2012. Effects of energy supplementation frequency and forage quality on performance, reproductive, and physiological responses of replacement beef heifers. J. Anim. Sci. 90:2371-2380. doi:10.2527/jas.2011-4958

NRC (National Research Council). 2016. Nutrient requirements of beef cattle. $7^{\text {th }}$ ed. National Academies Press, WA, USA.

Ørskov, E.R., D. Hovell, and F. Mould. 1980. The use of the nylon bag technique for the evaluation of feedstuffs. Trop. Anim. Prod. 5(3):195-203.

Rao, I., M. Peters, A. Castro, R. Schultze-Kraft, D. White, M. Fisher, J. Miles, C. Lascano, M. Blümmel, D. Bungestab, J. Tapasco, G. Hyman, A. Bolliger, B. Paul, R. Van-der-Hoek, B. Maass, T. Tiemann, M. Cuchillo, S. Douxchamps, C. Villanueva, Á. Rincón, M. Ayarza, T. Rosenstock, G. Subbarao, J. Arango, J. Cardoso, M. Worthington, N. Chirinda, A. Notenbaert, A. Jenet, A. Schmidt, N. Vivas, R. Lefroy, K. Fahrney, E. Guimarães, J. Tohme, S. Cook, M. Herrero, M. Chacón, T. Searchinger, and T. Rudel. 2015. LivestockPlus - The sustainable intensification of forage-based agricultural systems to improve livelihoods and ecosystem services in the tropics. Trop. Grassl. 3:59-82. doi:10.17138/TGFT(3)59-82

Reza-García, S., S. Mejía, H. Cuadrado, L. Torregroza, N. Jiménez, M. Espinoza, y G. Palencia. 2011. Experiencias en la implementación de modelos intensivos de producción de carne en pasturas fertirrigadas en el Valle del Sinú. CORPOICA, Cereté, COL.

Santana, A., C. Camacho, L. Estevés, M. Gómez, J. Gutiérrez, M. Rozo, y H. Ballesteros. 2009. Agenda prospectiva de la investigación y desarrollo tecnológico para la cadena cárnica bovina en Colombia. Ministerio de Agricultura y Desarrollo Rural. Bogotá, COL.

Senft, R.L., M.B. Coughenour, D.W. Bailey, L.R. Rittenhouse, O.E. Sala, and D.M. Swift. 1987. Large herbivore foraging and ecological hierarchies. BioScience 37:789-799.

Sossa, C., y R. Barahona. 2015. Comportamiento productivo de novillos pastoreando en trópico de altura con y sin suplementación. Rev. Med. Vet. Zootec. 62:67-80. doi:10.15446/rfmvz.v62n1.49386

Torregroza, L., M. Palomino, M. González, R. Salgado, y U. Gómez. 2004. Modelo de producción de dos toneladas de carne por hectárea año en el valle del Sinú. Revista MVZ-Córdoba 9:373-380. doi:10.21897/rmvz.505

Torregroza, L., S. Reza, E. Suárez, M. Espinosa, H. Cuadrado, I. Pastrana, y Y. Abuabara. 2015. Producción de carne en pasturas irrigadas y fertilizadas de Brachiaria híbrido cv. Mulato II en el valle del Sinú. CORPOICA Cienc. Tecnol. Agropecu. $16: 131-138$

Van-Soest, P.J., J.B. Robertson, and B.A. Lewis. 1991. Methods for dietary fiber, neutral detergent fiber, and nonstarch polysaccharides in relation to animal nutrition. J. Dairy Sci. 74:3583-3597. doi:10.3168/JDS.S0022-0302(91)78551-2

Velásquez, J.C., y L.A. Álvarez. 2004. Relación de medidas bovinométricas y de composición corporal in vivo con el peso de la canal en novillos Brahman en el valle del Sinú. Acta Agron. 53(3):61-68.

Viloria, J. 2003. La ganadería bovina en Colombia. Documentos de trabajo sobre economía regional. No. 40. Banco de la República, Cartagena de Indias, COL. http://www.banrep.gov.co/sites/default/files/publicaciones/archivos/DTSER-40. pdf (consultado 15 ene. 2019). ISSN 2215-3608 doi:10.15517/am.v31i2.38389 